Revue Française de Civilisation Britannique

XXII-3 | 2017

Forms of Activism in the United Kingdom (Grassroots Activism, Culture, Media)

\title{
L'Engagement des Niveleuses et la presse anglaise contemporaine (1649)
}

Leveller Women's Activism and the Contemporary English Press (1649)

\section{Laurent Curelly}

\section{CpenEdition}

Journals

Édition électronique

URL : http://journals.openedition.org/rfcb/1476

DOI : $10.4000 /$ rfcb. 1476

ISSN : 2429-4373

Éditeur

CRECIB - Centre de recherche et d'études en civilisation britannique

Référence électronique

Laurent Curelly, "L'Engagement des Niveleuses et la presse anglaise contemporaine (1649) », Revue Française de Civilisation Britannique [En ligne], XXII-3 | 2017, mis en ligne le 05 juillet 2017, consulté le 01 mai 2019. URL : http://journals.openedition.org/rfcb/1476 ; DOI : 10.4000/rfcb.1476

Ce document a été généré automatiquement le 1 mai 2019.

\section{c)}

Revue française de civilisation britannique est mis à disposition selon les termes de la licence Creative Commons Attribution - Pas d'Utilisation Commerciale - Pas de Modification 4.0 International. 


\title{
L'Engagement des Niveleuses et la presse anglaise contemporaine (1649)
}

\author{
Leveller Women's Activism and the Contemporary English Press (1649)
}

\author{
Laurent Curelly
}

\section{Introduction}

1 L'engagement des Niveleurs en faveur des libertés publiques et de l'émancipation politique du peuple est bien connu. Issu de la première guerre civile, qui déchira les îles Britanniques entre 1642 et 1646, ce groupe de radiaux œuvra continûment, aux côtés des soldats et des officiers de rang inférieur de l'Armée du Nouveau Modèle, à un règlement politique du royaume qui respectât les libertés ancestrales du peuple d'Angleterre, celles qu'il hérita censément de son passé anglo-saxon avant son asservissement dû à l'imposition du système féodal normand. À l'époque de la deuxième guerre civile en 1648, les Niveleurs militaient pour la mise en place d'un parlement renouvelé annuellement et représentatif, grâce à un suffrage élargi, et demandaient que soit adopté un document constitutionnel, l'Accord du peuple, auquel devaient souscrire tous les citoyens, en gage de leur participation pleine et entière à un régime démocratique débarrassé des scories de la monarchie Stuart. Les espoirs qu'ils avaient nourris suite à la défaite de Charles I lors de la deuxième guerre civile furent très tôt déçus, alors que la république nouvellement installée ne tenait pas ses promesses mais se transformait plutôt en oligarchie peu encline à tolérer les opposants au régime. Les Niveleurs combattirent ces dérives autoritaires dans des pamphlets souvent cinglants, fustigeant en particulier l'organe exécutif de la république, le Conseil d'État, ce qui leur valut d'être arrêtés, puis emprisonnés, début mars 1649. Le pamphlet qui mit le feu aux poudres, Englands New-Chaines Discovered, était une condamnation sans appel du joug que, selon ses auteurs, le régime imposait au peuple. Les Niveleurs mirent en récit leur arrestation, laquelle suscita une vague de pétitions de soutien, dont la presse se fit largement l'écho. 
2 En provenance de Londres mais aussi de la province, ces pétitions n'eurent guère d'effet immédiat, le Parlement Croupion et les dirigeants de la république se montrant sourds aux doléances de leurs signataires. Les Niveleurs ne furent pas libérés ; au contraire, les mesures de sécurité à la Tour de Londres furent renforcées et les visites strictement réglementées. En revanche, les pétitions demandant la libération de Lilburne et de ses amis en suscitèrent d'autres, permettant aux Anglais exclus du suffrage, en raison de leur statut social, de s'engager dans la campagne de mobilisation en faveur des Niveleurs emprisonnés et d'accéder ainsi à une forme de pouvoir politique. Quoiqu'à un degré moindre, les Niveleuses ${ }^{1}$ 'approprièrent elles aussi cette " politique participative $~^{2}$. Leur engagement est moins connu que celui de leurs époux ; pourtant, elles tentèrent de leur côté d'obtenir la libération de leurs maris en envoyant deux pétitions à la Chambre des communes. Il était tout à fait inédit que des femmes, fortes de l'appui de milliers de signataires, s'emparent de l'arme pétitionnaire et pénètrent de manière aussi insistante la sphère politique. Les pétitions pouvaient jusqu'alors être l'expression de voix féminines individuelles, rarement celle d'une voix collective, et encore moins porter des revendications politiques aussi sensibles.

3 C'est précisément l'engagement de ces femmes que, dans un premier temps, le présent article souhaite évaluer. Les journaux du printemps 1649 relatèrent leur démarche, tantôt pour la décrier, tantôt pour la vanter. Cette étude a également pour objet d'analyser la représentation "médiatique » de ce combat et, en particulier, de mettre en évidence le point de contact entre l'accaparement par les Niveleuses d'une forme de pouvoir politique et une certaine démocratisation de la presse, laquelle ne manquera pas d'être rapidement jugulée par les autorités de la république.

\section{L'engagement des Niveleuses}

4 Les Niveleuses présentèrent à la Chambre des communes deux pétitions, respectivement le 23 avril et le 7 mai 1649. La première pétition, qui aurait recueilli 10000 signatures et aurait été portée par plusieurs centaines de femmes ${ }^{3}$, réclamait la libération des Niveleurs, à l'instar d'autres pétitions contemporaines rédigées par des hommes. Elle épousait le combat mené par Lilburne, Overton, Walwyn et Prince en faveur de la défense des libertés fondamentales du peuple, que garantirait une nouvelle assemblée élue en lieu et place du Parlement Croupion, conformément aux revendications de la Grande Pétition du 11 septembre 1648 et aux dispositions de l'Accord du peuple ${ }^{4}$. Même si ceci n'est pas explicitement formulé dans la pétition, il s'agissait de mettre en place un système politique capable d'empêcher la confiscation des pouvoirs par une oligarchie, fût-elle républicaine. Les femmes demandaient aussi au Parlement d'appliquer avec davantage de mesure les condamnations pour trahison. Elles visaient en premier lieu la résolution que passa la Chambre des communes le 27 mars pointant du doigt le caractère subversif et dangereux de The Second Part of Englands New-Chains discovered, pamphlet rédigé par Lilburne et publié trois jours auparavant ${ }^{5}$.

5 Les femmes se virent refuser l'accès à la Chambre à deux reprises. Elles se montrèrent opiniâtres, et ce n'est que lors de leur troisième tentative, le 25 avril, que les députés leur prêtèrent attention, mais pour mieux les congédier. La réponse des Communes fut sans appel, mais sans surprise non plus, tant elle était révélatrice de l'ordre patriarcal dominant. On conseilla vivement aux femmes de s'en retourner à leurs foyers : 
You that are the women Petitioners, Mr Speaker, by direction of the House, hath commanded me to tell you, That the matter you Petition about is of an higher concernment then you understand, that the House gave an answer to your Husbands; and therefore that you are desired to goe home, and looke after your owne business and meddle with your huswifery ${ }^{6}$.

Les femmes obtempérèrent et quittèrent les lieux avec civilité, ainsi que le rapportèrent certains hebdomadaires ${ }^{7}$, la civilité toute teintée d'obéissance attendue d'elles eu égard à leur sexe. Si, dans un premier temps, elles se contentèrent de cette réponse et se soumirent à l'injonction qui leur était faite de regagner leurs foyers, c'est qu'elles avaient conscience du caractère transgressif de leur démarche. Elles dérogeaient ainsi aux règles patriarcales dominantes; même si elles s'étaient présentées au Parlement en tant qu'épouses dévouées, elles étaient entrées de plain-pied dans la sphère politique, parfaitement conscientes que leur engagement contrevenait aux normes sociales. C'est pourquoi, convoquant l'histoire biblique et le passé anglo-saxon de l'Angleterre en appui de leur démonstration, elles justifièrent leur intercession auprès de la Chambre des communes dans un préambule à leur pétition :

We are so over-prest, so over-whelmed in affliction, that we are not able to keep in our compass, to be bounded in the custom of our sex; for indeed we confess it is not our custom to address our selves to this House in the Publick behalf. [...] We know for our encouragement and example, God hath wrought many deliverances for severall Nations, from age to age by the weake hand of women ${ }^{8}$.

Ce premier manquement à l'ordre patriarcal contenait en germe une autre transgression, plus grave, laquelle s'exprima dans la seconde pétition que les femmes présentèrent au Parlement, le 5 mai 1649. L'objectif n'avait pas changé : il s'agissait en effet d'obtenir la libération de leurs époux, toujours prisonniers à la Tour de Londres. Entre-temps, ceux-ci avaient fait paraitre la troisième et dernière version de l'Accord du peuple, plus radicale que les deux premières car elle remettait en cause les institutions de la république; les Niveleurs revendiquaient une démocratie parlementaire libérée du Conseil d'État, organe exécutif du Commonwealth auquel ils devaient leur emprisonnement. Le printemps 1649 était marqué par des mutineries dans l'Armée suite à l'imposition d'un tirage au sort devant désigner les régiments susceptibles d'aller porter le fer en Irlande. Les soldats de l'Armée du Nouveau Modèle étaient donc sensibles aux thèses que défendaient les Niveleurs, et l'idée selon laquelle les dirigeants de la république faisaient peser sur le peuple un joug plus puissant que ne l'avaient fait les Stuart trouvait chez eux un réel écho.

8 Aussi, dans un contexte lourd de menaces pour l'intégrité du régime républicain, la deuxième pétition des Niveleuses, loin de n'être qu'un épiphénomène, symbolisait-elle le potentiel subversif de l'engagement politique, plus encore quand il était porté par des femmes. La pétition contient un hommage à Robert Lockyer, militant Niveleur de l'armée qui fut condamné pour trahison et exécuté, ainsi qu'un appel à l'indulgence de la Chambre des communes envers les Niveleurs. D'une tonalité sentimentale de nature à émouvoir les députés et empreint d'une rhétorique toute chrétienne, conformément aux convenances concernant la conduite et le langage des femmes, le message peine à dissimuler des intentions plus combatives, moins « féminines » :

Would you have us keep at home in our houses, when men of such faithfulnesse and integrity as the four prisoners our friends in the Tower, are fetcht out of their beds, and forced from their Houses by Souldiers, to the affrighting and the undoing of themselves, their wives, children and families? [...] Are we Christians, and shall we sit still and keep at home, while such men as have borne continual testimony 
against the unjustice of all times, and unrighteousnesse of men, be pickt out and be delivered up to the slaughter, and yet must we shew no sence of their sufferings, not tendernesse of affections, no bowels of compassion, nor bear any testimony against so abominable cruelty and injustice?

9 Les Niveleuses prirent donc la défense de leurs époux, au sens juridique du terme, et se référèrent en particulier aux Institutes du juriste Coke ainsi qu'à la Pétition de Droit de 1628, texte qui - comme on le sait - devait servir d'armature constitutionnelle à la monarchie parlementaire issue de la révolution de 1688. Les femmes utilisaient les mêmes arguments que leurs maris, ceux dont Lilburne, notamment, avait coutume de se servir, car il avait eu maille à partir avec la justice de son pays dont il avait dénoncé en plusieurs occasions le fonctionnement arbitraire. Toutefois, elles ne manifestèrent guère la retenue attendue d'elles en affirmant l'égalité naturelle des hommes et des femmes. Elles demandaient en effet dans la pétition un droit politique, celui de présenter des pétitions au Parlement, sur la base d'un droit naturel, les femmes ayant été conçues à l'image de Dieu et étant donc égales aux hommes en vertu de leur appartenance à l'espèce humaine. Le Parlement leur avait refusé ce droit quelques semaines auparavant; elles s'étaient alors montrées dociles mais elles s'affranchissaient désormais de leur réserve :

Since we are assured of our Creation in the image of God [...], equal unto men, as also of a proportionable share in the Freedoms of this commonwealth, we cannot but wonder and grieve that we should appear so despicable in your eyes, as to be thought unworthy to Petition or represent our Grievances to this Honourable House $^{10}$.

10 L'égalité que revendiquaient ici les Niveleuses se limitait à un aspect de la vie publique, celui de signer et de porter des pétitions, et d'ailleurs il n'était pas formellement interdit aux femmes de le faire. Elles ne se seraient pas aventurées, par exemple, à réclamer le droit de vote, l'eussent-elles voulu, quand, de toute façon, leurs époux militaient pour un suffrage quasi universel mais exclusivement masculin. En revanche, elles défendaient bel et bien un droit politique en s'inspirant du discours jus-naturaliste de leurs époux. C'était là déjà défier l'autorité masculine de la Chambre des communes, d'autant plus qu'il s'agissait d'un sujet sensible, le Parlement s'étant employé à restreindre les pétitions au sein de l'armée.

11 Elles franchissaient le Rubicon en déclarant la guerre - à tout le moins dans les mots - à la Chambre des communes, faisant fi du caractère policé qu'on leur prêtait :

Nor will we ever rest until we have prevailed, that We, our Husbands, Children, Friends, and Servants, may not be liable to be thus abused, violated and butchered at mens Wills and Pleasures. But if nothing will satisfie but the bloud of those just men, those constant undaunted Asserters of the Peoples Freedoms will satisfie your thirst, drink also, and be glutted with our bloud, and let us fall together: Take the bloud of one more, and take all. Slay one, slay all ${ }^{11}$.

Il était peu vraisemblable qu'elles mettent leurs menaces à exécution, si tant est qu'elles en aient eu les moyens. Il n'en demeure pas moins que, empruntant à une rhétorique sacrificielle, elles pointaient un doigt vengeur en direction de ceux qui, selon elles, opprimaient leurs maris et, par extension, le peuple libre d'Angleterre. Elles s'emparaient donc du débat public avec véhémence.

On observe entre les deux pétitions présentées par les Niveleuses une escalade rhétorique : dans le second texte, le propos est comminatoire, voire agressif, et témoigne d'une prise de conscience aiguë des enjeux liés à l'arme pétitionnaire. L'engagement de ces femmes aux côtés de leurs époux participe de la radicalisation politique qui s'opère au 
printemps 1649 , le but recherché par les signataires étant de susciter une mobilisation contre le Parlement Croupion et le Conseil d'État, résistance pouvant, entre autres modalités, prendre la forme de mutineries dans l'armée.

Il est malaisé de définir la nature d'un tel engagement : s'agit-il d'une riposte ponctuelle au harcèlement dont étaient victimes les Niveleurs? Relève-t-il plus largement d'un combat d'idées dans lequel les femmes jouaient le rôle de porte-voix au service du programme politique de leurs époux ? Symbolise-t-il une forme d'affranchissement de la domination masculine s'exprimant par des revendications "proto-féministes »? Ann Hughes rejette toute vision téléologique qui ferait des Niveleuses les devancières des combattantes féministes du premier vingtième siècle, invitant à considérer les pétitions de ces femmes comme une modalité parmi d'autres du mouvement niveleur plutôt que comme une forme de militantisme féministe ${ }^{12}$. Elle prend le contrepied de H. N. Brailsford qui décrit les Niveleuses comme des pionnières, soutenues en cela par leurs maris, militants éclairés et en avance sur leur temps ${ }^{13}$. Dans une étude récente, Rachel Foxley reprend les thèses d'Ann Hughes, affirmant que les Niveleuses étaient pleinement conscientes des présupposés régissant les rapports entre les sexes dans l'Angleterre de la première modernité, et qu'en respectant le modèle patriarcal, elles s'assuraient une certaine forme de respectabilité ${ }^{14}$. On pourra reprocher à Brailsford le fait que ses arguments sont sous-tendus par une construction idéologique héritée de la pensée marxiste et qu'il applique sa croyance en une société égalitaire au cadre sexué normé de l'Angleterre du dix-septième siècle. Pour autant, Ann Hugues décrit les pétitions des Niveleuses comme "the climax of female, even feminist activism unleashed by the political upheavals of the 1640s", laissant entendre que l'intervention de ces femmes était à la fois singulière mais prévisible dans un contexte propice à l'épanouissement des expressions radicales ${ }^{15}$.

15 Fin janvier 1649 était publié The New Law of Righteousness, traité dans lequel Gerrard Winstanley, futur meneur des Bêcheurs («Diggers ») à St George’s Hill, dans le Surrey, exposait pour la première fois sa vision d'un monde idéal qui aurait aboli la propriété privée. Les femmes devaient y avoir toute leur part et pouvaient bénéficier au même titre que les hommes de la communauté des biens. Certes, ce texte n'était pas exempt de remarques misogynes, que Winstanley justifie en convoquant les Saintes Écritures, en particulier les écrits paulininens : les femmes symbolisent la corruption de la chair tandis que l'Esprit de la justice se manifeste dans le Christ, puissance masculine. Nonobstant, une fois la cupidité et la tentation du profit éliminées, hommes et femmes participent à égalité à la communauté régénérée qu'entrevoit Winstanley ${ }^{16}$. La loi de justice ( law of Righteousness ") qu'il décrit est fortement empreinte de son mysticisme chrétien, et l'on ignore quel rôle jouaient les femmes dans la petite communauté agraire du Surrey. Toutefois, l'égalité naturelle entre les deux sexes qu'il suggère n'est guère éloignée de celle que revendiquent les Niveleuses dans leur seconde pétition. Elles en font une arme politique, ce que n'envisage absolument pas Winstanley. On voit bien cependant que, par les pamphlets de Winstanley et des Bêcheurs comme par les pétitions des Niveleuses, ce sont des idées et des revendications qui s'expriment et s'agrègent comme autant d'énergies radicales suscitées par les soubresauts politiques des années 1640, pour reprendre la formule d'Ann Hughes. C'est à la lumière de ces expressions contestataires ${ }^{17}$ que doit être appréhendé l'engagement des Niveleuses au printemps 1649, engagement qui fut relayé, voire encouragé, par la presse contemporaine. 


\section{Les Niveleuses et la presse}

16 Si les journaux de l'époque se ressemblaient physiquement, ils reflétaient des opinions différentes, rendant ainsi compte des fractures politiques qui divisaient l'Angleterre. Les hebdomadaires de tradition parlementaire, en particulier ceux qui étaient établis de longue date, soutenaient le régime républicain, quand bien même ils avaient manifesté leur préférence pour les Presbytériens contre les Indépendants lors des discussions sur le Traité de Newport. Leur parution était soumise à l'imprimatur des censeurs et, s'ils souhaitaient poursuivre leurs activités et continuer d'engranger les dividendes de leur commerce, ils n'avaient pas intérêt à s'aliéner les dirigeants du Commonwealth. Les journaux royalistes, quant à eux, étaient publiés clandestinement, et leurs auteurs ne ménageaient pas leurs efforts afin d'éviter la saisie de leurs presses. Ils brocardaient volontiers le Parlement Croupion et le Conseil d'État, usant d'un ton le plus souvent irrévérencieux et polémique. Ces publications satiriques étaient des armes de propagande, qui visaient à attiser la colère des opposants au régime, afin que ceux-ci s'emparent d'armes bien réelles cette fois pour renverser la république et rétablir la monarchie. Les mécontents se trouvaient également dans les rangs de l'Armée du Nouveau Modèle ainsi qu'au sein des groupes radicaux, les Niveleurs notamment: les uns et les autres se sentaient floués et reprochaient aux autorités du Commonwealth d'avoir trahi la cause du peuple. De rares journaux relayaient leurs préoccupations, dont l'hebdomadaire radical The Moderate.

Ces publications diffusèrent dans leurs colonnes l'information relative aux pétitions des Niveleuses, leur prêtant plus ou moins d'attention, accompagnant parfois de commentaires la reproduction des textes. La plupart des journaux «autorisés» se contentèrent de publier la première pétition, jugeant probablement la seconde trop subversive pour la reproduire. Le risque que leurs auteurs auraient encouru en la diffusant était grand: c'eût été contrevenir à la loi de 1643 sur la presse et, vraisemblablement, se voir interdire la parution du numéro incriminé ou même du journal. Les rédacteurs devaient trouver un équilibre entre la nécessité de publier des nouvelles le plus rapidement possible afin de conserver leur lectorat et une forme de prudence face à des informations sensibles. C'étaient ces considérations qui dictaient leurs choix éditoriaux : les pétitions étaient porteuses d'un contenu essentiel, tant elles reflétaient une actualité immédiate, souvent brûlante et pouvant donc être saisie sur le vif. Il fallait veiller cependant à ne pas franchir la ligne rouge qui aurait mis le journal en difficulté. Ainsi peut-on interpréter le choix que firent certains hebdomadaires, non seulement de publier la première pétition des Niveleuses, mais aussi de relater les déboires que subirent ces femmes et la fin de non-recevoir qui leur fut opposée par les députés ${ }^{18}$. Ces reportages donnent des indications précieuses relatives à la chronologie des événements ainsi qu'aux modalités de diffusion des pétitions dans la sphère radicale. On apprend ainsi que la pétition des Niveleuses a circulé la veille de sa présentation au Parlement, lors des rassemblements congrégationalistes du dimanche dans la cité de Londres, mais aussi hors des murs de la ville, et qu'elle a fait l'objet de débats au sein des congrégations ${ }^{19}$. On mesure ainsi les liens intimes unissant le radicalisme religieux et le radicalisme politique dans l'Angleterre du dix-septième siècle, sans qu'il y ait nécessairement d'adéquation totale entre ces deux formes radicales. Ce n'est pas un hasard si, dans une pétition qu'ils adressèrent à la Chambre des communes le 2 avril 1649, 
des "Anabaptistes ", ainsi qu'ils se nommèrent, rejetèrent tout amalgame avec les Niveleurs, qui venaient de publier leur pamphlet incendiaire Second part of Englands NewChaines discovered ${ }^{20}$. Il s'agissait pour eux de ne pas subir le même sort que les Niveleurs mais aussi de se prévaloir d'une forme d'intégrité morale garante de leur obéissance au nouvel ordre politique qui suivit le régicide.

C'est vraisemblablement parce qu'elle menaçait cet ordre que la seconde pétition des Niveleuses fut généralement passée sous silence dans la presse parlementaire. Seuls deux journaux établis relatèrent l'événement, de manière neutre, sans reproduire le texte ${ }^{21}$, cependant que deux autres hebdomadaires, récemment installés, pimentèrent leur compte-rendu de la démarche des Niveleuses de commentaires phallocrates incisifs visant à discréditer ces femmes. Ainsi, A Modest Narrative of Intelligence évoque les Niveleuses rassemblées devant le Parlement, pétition en main et arborant des rubans verts en hommage à leurs époux et amis enfermés à la Tour de Londres ainsi qu'au soldat Lockyer, devenu martyr de la cause Niveleuse. Le rédacteur conclut sa dépêche par ces mots : « They have ever loved to meddle with what they would not from the beginning; small baits will soon catch such silly fish ${ }^{22}$. " De la même manière, The Impartiall Intelligencer rapporte cette anecdote: "A Gentleman told them, That if they understood themselves they might be better occupied at home ${ }^{23}$ ", peinant à masquer son propre jugement. Ces deux journaux avaient déjà tourné en dérision la première pétition des Niveleuse, en particulier The Impartiall Intelligencer, qui s'acharna sur elles, balayant leur plainte d'un trait de plume sentencieux: "That is as much as to say, Let Women weare the breeches [...] It can never be a good world, when women meddle in State matters. [...] We shall have things brought to a fine passe, if women come to teach the Parliament how to make Lawes ${ }^{24}$."

Soucieux de de leur image de sérieux les hebdomadaires parlementaires ayant acquis une réputation de longue date s'en tinrent prudemment à une fausse neutralité, relayant l'information relative aux pétitions des Niveleuses, principalement la première, sans toutefois omettre la réponse des députés qui déboutaient ces femmes et les renvoyaient chez elles. Les publications éphémères, quant à elles, adoptèrent pour cet événement un ton caustique digne des journaux royalistes de l'époque. La préservation d'une forme de respectabilité sociale valait bien que l'on jetât l'opprobre sur les Niveleuses et l'on employât un ton polémique à leur endroit. On ne saurait sous-estimer la perception d'un événement apparemment anodin, mais hautement symbolique, dans un pays où les structures politiques liées à la monarchie s'étaient effondrées avec elle et où l'ordre social risquait lui aussi d'être mis à mal. À la même époque, les Bêcheurs suscitaient l'intérêt de la presse : moqués dans un premier temps, ils furent ensuite pris au sérieux, avant qu'ils ne soient dispersés et que l'emballement «médiatique " ne retombe. Les autorités du Commonwealth, dont les journaux se faisaient l'écho, craignaient que ces mouvements d'opposition sporadiques ne s'agrègent, comme l'écrivit John Bradshaw, le Président du Conseil d'État, dans une missive adressée à Fairfax, commandant en chef de l'armée, justifiant ainsi la répression menée contre les Bêcheurs : «To prevent the like for the future, that a malignant and disaffected partie may nott under colour of such ridiculous people have any opportunity to rendez-vous themselves in order to a greater mischief ${ }^{25}$. " On perçoit à quel point ces épiphénomènes qu'étaient la constitution de la petite colonie des Bêcheurs et les pétitions des Niveleurs étaient jugés transgressifs et faisaient craindre un bouleversement des soubassements sociaux et moraux de l'Angleterre. royaliste après le régicide offre un éclairage décentré sur les événements contemporains, 
à condition de ne pas occulter le caractère polémique des journaux. Leurs auteurs y expriment leur engagement inconditionnel en faveur du prince Charles - le futur Charles II - et, plus largement, de la monarchie, dont ils demeurent convaincus qu'elle renaitra de ses cendres. L'exécution du roi Charles I avaient certes provoqué une telle sidération qu'elle les avait poussés au silence, du moins temporairement. En revanche, au moment où les Niveleuses présentaient leurs pétitions au Parlement, les journaux royalistes étaient de nouveau en pleine éclosion. Ils n'avaient pas perdu leur verve satirique ; bien au contraire, ils s'employaient à fustiger les institutions de la république, tant le Parlement Croupion que le Conseil d'État, versant dans l'invective, voire dans la grivoiserie. The Man in the Moon, par exemple, n'économisait pas ses critiques envers le Commonwealth, usant fréquemment de comparaisons scatologiques. On peut s'étonner de la vitalité de la presse royaliste au printemps 1649 alors que le régicide avait provoqué l'effroi. Ces écrits permettaient aux auteurs de maintenir vivace une culture littéraire de cour, à laquelle ils empruntaient volontiers, et, alors que la légitimité du nouveau régime était contestée, en particulier par les radicaux, de susciter l'espoir d'une contrerévolution. Souffler sur les braises du mécontentent, inciter les déçus de la république et ses opposants à se fédérer, tel était l'objectif, parfois avoué, des journaux royalistes.

21 Il n'est guère surprenant, dans ce contexte, que ceux-ci se soient emparés de l'affaire des Niveleuses, dont ils relatèrent les déboires et à qui ils apportèrent leur soutien. C'était un soutien de circonstance; les Niveleurs avaient souvent été l'objet de moqueries et de critiques dans leurs colonnes, mais il fallait faire feu de tout bois. Les alliances étaient volatiles, et les Niveleurs eux-mêmes se rapprochèrent des royalistes. Dans leurs reportages sur les Niveleuses, les auteurs recoururent à quelques clichés misogynes, s'amusant en particulier de la loquacité supposée des femmes ou du jeu de séduction auquel elles se livrèrent auprès des députés ${ }^{26}$. En revanche, leurs comptes rendus ne sont pas dépourvus de sympathie, en particulier quand ils soulignent le mépris avec lequel le Parlement les aurait congédiées. Ces guerrières en jupon - ces "Troopes of Amazons ", comme l'écrit Mercurius Pragmaticvs - sont présentées comme des figures héroïques qui, malgré leur impuissance à faire entendre leurs griefs, se montrèrent à ce point valeureuses qu'elles remportèrent une bataille symbolique contre les députés en les ridiculisant. Les hebdomadaires royalistes saluent la combativité de ces femmes et légitiment ainsi leur démarche: les Niveleuses deviennent les porte-drapeaux d'une liberté bafouée par le Commonwealth. Les rédacteurs de ces journaux se jouent des codes sociaux patriarcaux, la fin - c'est-à-dire le renversement de la république et le rétablissement de la monarchie - justifiant les moyens.

Étonnamment, seule une des publications royalistes évoqua la seconde pétition des Niveleuses. Peut-être son auteur avait-il un informateur au Parlement ou peut-être les rédacteurs des autres journaux se ravisèrent-ils, préférant ne pas cautionner l'engagement « féministe » des Niveleuses. Il est impossible de le dire, tant l'information véhiculée dans les hebdomadaires royalistes dépendait des conditions matérielles de production de ces publications : il fallait souvent rédiger les reportages à la hâte afin d'éviter que les presses ne soient découvertes ${ }^{27}$. Toujours est-il que Mercurius Pragmaticus (for King Charles II) décrit la présentation avortée de la seconde pétition au Parlement comme une fable dont la morale ne laisse aucun doute sur l'issue pressentie du combat des Niveleuses: "They shall know what it is to jeer at Woemen with their Huswifry, when their businesse is liberty ${ }^{28}$.» 

liberté qu'elles défendaient se caractérisait par son opportunisme. Il était en effet peu concevable que ses auteurs se soient soudain enthousiasmés pour les revendications démocratiques que portaient les Niveleurs - suffrage quasi universel et souveraineté du peuple s'incarnant dans un mode de gouvernement contractualiste. De leur côté, les Niveleurs se ralliaient à l'idée qu'une monarchie mixte valait mieux qu'une république oligarchique et autoritaire. Aussi l'intérêt des journaux royalistes pour les pétitions des Niveleuses s'explique-t-il non pas par une adhésion spontanée à leur démarche mais par l'espoir que ces munitions de papier se transforment en armes bien réelles, quand tous les mécontentements se fédèreraient, et contribueraient à la mise en échec du Commonwealth. Mercurius Pragmaticvs en appelle clairement à la sédition, pointant du doigt les insuffisances de l'arme pétitionnaire : «If you do nothing but Petition, you will prove that words are but wind and will worke you to no good, but rather provoke your Enemies more to hatred; therefore be bold, act something, there are Women enough to destroy Cromwell's faction. [...] Leave Petitioning, draw your Swords, and try for your freedomes, dispute them to the chin in blood $^{29}$. » Il revenait donc aux Niveleuses d'être le fer de lance d'une contre-révolution qui rassemblerait tous les mouvements de résistance à la république. journaux radicaux qu'étaient Mercurius Militaris et The Moderate. Le premier reparut, pour quelques numéros seulement, en avril et mai 1649, après un long silence. Il s'agissait d'un journal éphémère qui fut publié à deux reprises, d'abord à l'automne 1648, puis au printemps 1649, et qui accompagna deux épisodes importants de ces années charnières dans l'histoire des guerres civiles britanniques, lors desquels l'Armée s'imposa comme acteur politique majeur : respectivement, la mobilisation des soldats contre le Traité de Newport, largement soutenu par le Parlement, et leur résistance à l'envoi des régiments en Irlande, qui se traduisit par des mutineries et s'exprima dans un contexte d'opposition grandissante au pouvoir en place. Dans la veine satirique des journaux royalistes, Mercurius Militaris manifesta un soutien entier aux pétitions des Niveleuses tant son auteur approuvait l'affront ainsi fait aux dirigeants de la république : déplorant l'accueil peu courtois et inhumain ("uncivill and inhumane ») qui leur fut réservé quand elles se présentèrent la première fois auprès de la chambre des Communes $^{30}$, il adopta un ton nettement plus vindicatif quand il évoqua des pétitions ultérieures en faveur de l'élargissement des Niveleurs emprisonnés, dont celle de leurs épouses. Cet épisode lui fournit matière à pourfendre Cromwell et ses affidés dans une diatribe violente, dont les Niveleuses devenaient d'infortunées auxiliaires : «The women are marching down in Batalia to give the members of Westminster a second charge, with the artillery of a Petition. [...] But Nol and his asses / Do feare the brave lasses / And therefore begin to fle ${ }^{31}$ ".

The Moderate fut le seul journal à reproduire la seconde pétition des femmes dans son intégralité $^{32}$. D'autres pétitions de soutien aux Niveleurs emprisonnés avaient trouvé droit de cité dans le même numéro. Les pétitions n'étaient pas assorties de commentaires éditoriaux alors que d'autres rubriques, en particulier les comptes rendus des travaux du Parlement, étaient émaillées de remarques incisives dont les thèmes étaient pour l'essentiel empruntés aux Niveleurs. Il convient cependant de considérer le numéro du journal comme un tout; même si les nouvelles étaient présentées de manière kaléidoscopique, elles comportaient de nombreux échos et résonances intra-textuelles de nature à guider la lecture. L'engagement des femmes pour leurs époux mais aussi pour elles-mêmes, dans la mesure où il sous-tendait une forme d'émancipation politique,

Revue Française de Civilisation Britannique, XXII-3 | 2017 
représentait tout ensemble une remise en cause de l'ordre patriarcal et une menace immédiate pour la république. Rendant compte de cette mobilisation et lui apportant leur soutien, les journaux royalistes comme les journaux radicaux se rendaient complices de ces actes de déstabilisation de la république.

\section{Conclusion}

26 Ce n'est pas un hasard si les divers hebdomadaires de l'époque se firent souvent l'écho de pétitions. Armes politiques, celles-ci pouvaient en effet donner lieu à une surenchère, entrainant la publication d'autres pétitions dans leur sillage. C'est dire qu'elles possédaient un potentiel de contestation politique évident ainsi qu'une valeur commerciale non négligeable. Cette forme de discours se radicalisa dans les années 1649-1650 au gré des soubresauts qui agitèrent l'Angleterre et des prises de position des différents acteurs ${ }^{33}$. L'engagement des Niveleuses participait certes d'une démarche politique visant à obtenir la libération de leurs époux, mais il permettait également à ces femmes en tant qu'êtres sexués de contester les normes sociales en vigueur dont elles se sentaient victimes. En publiant leurs deux pétitions, une certaine presse leur offrait une tribune, ajoutant leurs revendications à celles d'autres groupes radicaux. Porter la voix des opprimés à une époque où s'agrégeaient les énergies radicales: tel était son l'engagement. Si les rédacteurs des journaux royalistes étaient motivés par un opportunisme à peine masqué, les auteurs des hebdomadaires radicaux avaient pour dessein de dénoncer un ordre politique et social qu'ils jugeaient attentatoire aux libertés du peuple. D’un point de vue méthodologique, l'étude de ces écrits éphémères (« cheap print ») qu'étaient les pétitions et les journaux dans l'Angleterre de la première modernité permet de mieux saisir comment l'engagement des Niveleuses aux côtés de leurs époux se mua en une forme de participation politique; elle permet également de mesurer le soutien que reçurent ces femmes ainsi que l'opposition à laquelle elles se heurtèrent. Semblable étude nous invite à réhabiliter cette histoire des marges que l'historiographie marxiste avait promue, de manière parfois trop systématique, mais que nombre d'historiens de la génération suivante ("revisionist historians ») mirent sous l'éteignoir. Au confluent de divers champs disciplinaires, l'approche croisée que favorise l'étude des modes de diffusion des idées radicales ouvre pourtant des perspectives prometteuses, propices notamment à une réévaluation de la nature et des formes de l'engagement porté par des groupes « mineurs » pendant les guerres civiles anglaises.

Laurent Curelly est Maître de Conférences à l'Université de Haute Alsace (Mulhouse) et membre du laboratoire ILLE (Institut de Recherche en Langues et Littératures Européennes, EA 4363). Pluridisciplinaires, situées au carrefour de l'histoire politique, de l'histoire des idées et de la littérature, ses recherches portent principalement sur la presse anglaise des années révolutionnaires (1642-1660) et sur les mouvements radicaux à la même époque. Il est l'auteur de nombreux articles sur ces sujets et a récemment publié, avec Nigel Smith, un ouvrage collectif intitulé Radical Voices, Radical Ways - Articulating and Disseminating Radicalism in Seventeenthand Eighteenth-Century Britain (Manchester University Press, 2016). 


\section{BIBLIOGRAPHIE}

Sources primaires

A Modest Narrative of Intelligence, $\mathrm{n}^{\circ}$ 6, 5-12 mai 1649.

A Perfect Diurnall, n³00, 23-30 avril 1649.

Continued Heads of Perfect Passages in Parliament n², 20-27 avril 1649.

House of Commons Journal, vol. 6, 27 mars 1649.

Kingdomes Faithfull and Impartial Scout, n¹3, 20-27 avril 1649.

Mercurius Elenticus, n¹, 24 avril - 1 mai 1649.

Mercurius Militaris, Or The People's Scout, n¹, 17-24 avril.

Mercurius Militaris, Or The People's Scout, n³, s.d.

Mercurius Pragmaticvs, n52, 24 avril - 1 mai 1649.

Mercurius Pragmaticus (for King Charles II), n³, 1-8 mai 1649.

Perfect Occurrences, ${ }^{\circ} 121,20-27$ avril 1649.

Perfect Occurrences, $\mathrm{n}^{\circ} 123,4-11$ mai 1649.

Perfect Weekly Account, non numéroté, 18-25 avril 1649.

The humble Petition of divers well-affected Women Inhabiting the Cities of London and Westminster, the borough of Southwark, Hamblets [sic] and Places Adjacent. Affecters and Approvers of the late large Petition of the Eleventh of September 1648, Londres, 1649.

The humble Petition and Representation of severall churches of God in London, commonly, though falsely, called Anabaptists, Londres, 1649.

The Humble Petition of divers well-affected women, of the Cities of London and Westminster, the Borough of Southwark, Hamblets [sic] and Parts Adjacent. Affecters and Approvers of the Petition of Sept. 11. 1648, Londres, 1649.

The Impartiall Intelligencer, $\mathrm{n}^{\circ} 8,18-25$ avril 1649.

The Impartiall Intelligencer, $\mathrm{n}^{\circ}$ 10, 2-9 mai 1649.

The Kingdomes Weekly Intelligencer, ${ }^{\circ} 309,24$ avril - 1 mai 1649.

The Kingdomes Weekly Intelligencer, $n^{\circ} 310,1-8$ mai 1649.

The Moderate: Impartially communicating Martial Affaires to the Kingdom of England, $\mathrm{n}^{\circ} 43,1-8$ mai 1649.

WINSTANLEY Gerrard, « The New Law of Righteousness ", in The Complete Works of Gerrard Winstanley. CORNS Thomas N., HUGHES Ann et LOEWENSTEIN David (dir.), Oxford, Oxford University Press, 2009, vol. 1, p. 472-600.

Sources secondaires

BRAILSFORD H. N., The Levellers and the English Revolution (1961), Nottingham, Spokesman, 1983. 
CURELLY Laurent, "'Our Digging upon that common is the Talk of the Whole Land' : The Story of the Surrey Diggers as Told by Contemporary Newsbooks », in BANDRY-SCUBBI Anne et CHARDIN Jean-Jacques (dir.), Diffusion de l'écrit dans le monde anglophone-Spreading the Written Word in the English-Speaking World, Revue de la Société d'Études Anglaises et Américaines XVII-XVIIIe siècles, H.S. n 2, 2010, pp. 47-62.

FOXLEY Rachel, The Levellers - Radical Political Thought in the English Revolution, Manchester, Manchester University Press, 2013.

HOLSTUN James, Ehud's Dagger - Class Struggle in the English Revolution, London \& New York: Verso, 2000.

HUGHES Ann, « Gender and politics in Leveller literature », in AMUSSEN Susan D. et KISHLANSKY Mark (dir.), Political Culture and Cultural Politics in Early Modern England, Manchester, Manchester University Press, 1995, p. 162-188.

LUTAUD Olivier, Winstanley - Socialisme et christianisme sous Cromwell, Paris, Didier, 1976.

MCELLIGOTT Jason, Royalism, Print and Censorship in Revolutionary England, Woodbridge, The Boydell Press, 2007.

PEACEY Jason, « The parliamentary context of political radicalism in the English revolution », in CURELLY Laurent et SMITH Nigel, Radical Voices, Radical Ways - Articulating and Disseminating Radicalism in Seventeenth- and Eighteenth-Century Britain, Manchester, Manchester University Press, 2016, p. 151-69.

PEACEY Jason, Print and Public Politics in the English Revolution, Cambridge, Cambridge University Press, 2013.

ZARET David, Origins of Democratic Culture - Printing, Petitions, and the Public Sphere in Early-Modern England, Princeton, Princeton University Press, 2000.

\section{NOTES}

1. Nous revendiquons l'étiquette "Niveleuses» en référence aux épouses des Niveleurs. Leur engagement est décrit dans le présent essai ; aussi est-il naturel que la féminisation du nom « Niveleurs » s'applique à elles. La question se pose avec moins d'acuité en anglais, qui utilisera le terme de "Leveller women» ou "female Levellers", cependant que l'appellation générique «Levellers » désigne presque toujours les hommes.

2. Nous reprenons ici le terme de "participatory politics ", utilisé par Jason PEACEY dans Print and Public Politics in the English Revolution, Cambridge, Cambridge University Press, 2013. Son analyse de la pétition en tant que forme de participation politique emprunte à la thèse développée par David zaReT dans Origins of Democratic Culture - Printing, Petitions, and the Public Sphere in EarlyModern England, Princeton, Princeton University Press, 2000.

3. Le chiffre concernant les signataires est donné par le journal radical Mercurius Militaris, Or The People's Scout, n¹, 17-24 avril 1649, p. 13. L'estimation du nombre de femmes qui présentèrent la pétition au Parlement varie d'un journal à l'autre : les femmes auraient été plusieurs centaines, selon Perfect Occurrences n ${ }^{\circ} 121,20-27$ avril 1649, p. 993 ; «trois cents », d'après Kingdomes Faithfull and Impartial Scout $n^{\circ} 13,20-27$ avril 1649, p. 101 ; «cinq cents ", à en croire Continued Heads of Perfect Passages in Parliament n², 20-27 avril 1649, p. 11 ; voire " une multitude », comme l'écrit Perfect Weekly Account, non numéroté, 18-25 avril 1649, p. 460. L'ordre de grandeur suffit à montrer la portée de la démarche des femmes. Comme aujourd'hui, l'utilisation des chiffres 
pouvait nourrir des intentions polémiques et/ou flatter le goût du public pour le spectaculaire et le sensationnel.

4. La Grande Pétition de septembre 1648 marqua l'histoire des Guerres Civiles anglaises dans la mesure où, en elle, s'agrégèrent les revendications des Niveleurs et celles de l'Armée du Nouveau Modèle. Au premier rang de ces demandes pressantes figurait le rejet de toute négociation avec le roi et du compromis que représentait le Traité de Newport, souhaité par la majorité presbytérienne au Parlement. Cette pétition tutélaire en entraîna d'autres dans son sillage, lesquelles s'en réclamèrent et la citèrent abondamment. L'Accord du peuple (Agreement of the People ) était un projet de constitution qui avait les faveurs des Niveleurs. Fermement discuté à Putney à l'automne 1647 , ce texte, connut plusieurs moutures. La pétition des femmes se réfère à la deuxième version, celle de l'hiver 1648 ; les Niveleurs en publièrent une troisième depuis la Tour de Londres le $1^{\text {er }}$ mai 1649.

5. House of Commons Journal, vol. 6, 27 mars 1649, p. 175.

6. «À vous les femmes qui présentez cette pétition, j'ai été chargé de dire par Monsieur le Président, lui-même mandaté par la Chambre, que le sujet de votre pétition concerne des questions que vous n'êtes pas en mesure de comprendre, que la Chambre a déjà répondu à vos époux, et que, par conséquent, il vous est demandé de rentrer chez vous, de vous occuper de vos affaires et de vous en tenir à vos tâches domestiques. "Cette déclaration comminatoire fut reprise dans plusieurs journaux, dont: A Perfect Diurnall n³00, 23-30 avril 1649, p. 2439 (pagination irrégulière); The Kingdomes Weekly Intelligencer $n^{\circ} 309,24$ avril - 1 mai 1649, p. 1339 ; Perfect Occurrences n ${ }^{\circ} 121,20-27$ avril 1649, p. 998.

7. Voir, par exemple, The Kingdomes Weekly Intelligencer $n^{\circ} 309$, p. 1339, ou Perfect Occurrences $n^{\circ}$ 121, p. 998.

8. «Nos cœurs sont à ce point affligés et écrasés de chagrin que nous ne pouvons pas rester cantonnées dans notre sphère ni demeurer prisonnières des traditions dévolues à notre sexe. Et, de fait, nous reconnaissons qu'il n'est pas dans nos habitudes de porter une parole publique auprès de la Chambre. [...] Pour nous encourager, nous savons qu'il existe des exemples de nations libérées, sous l'action de Dieu, par la main de faibles femmes. " The humble Petition of divers well-affected Women Inhabiting the Cities of London and Westminster, the borough of Southwark, Hamblets [sic] and Places Adjacent. Affecters and Approvers of the late large Petition of the Eleventh of September 1648, Londres, 1649, p. 4.

9. «Voudriez-vous que nous restions chez nous alors que nos quatre amis incarcérés à la Tour de Londres, hommes d'une si haute loyauté et d'une si grande probité, sont arrêtés en pleine nuit par des soldats qui les obligent à quitter leur maison, apeurés, sous le regard terrifié de leurs femmes, de leurs enfants et de leurs familles ? [...] Serions-nous chrétiennes si nous restions sans bouger chez nous, alors que ces hommes qui n'ont cessé de témoigner contre l'injustice et la méchanceté des hommes de tout temps, sont cueillis et conduits à la boucherie? Devrions-nous rester insensibles à leurs souffrances, ne manifester ni affection ni compassion en ouvrant nos cœurs et nos entrailles, et ne pas rendre témoignage d'une cruauté et d'une injustice si abominables?» The Humble Petition of divers well-affected women, of the Cities of London and Westminster, the Borough of Southwark, Hamblets [sic] and Parts Adjacent. Affecters and Approvers of the Petition of Sept. 11. 1648, Londres, 1649, p. 1. Le titre de cette pétition ne diffère que marginalement de celui de la première. La pétition est datée du 5 mai par Thomason. Comme le montre l'extrait, elle est imprégnée de références bibliques.

10. «Nous avons la certitude d'avoir été créées à l'image de Dieu [...], d'être égales aux hommes et d'avoir droit à une part équitable des libertés de cette république. Nous ne pouvons donc que nous étonner et déplorer d'apparaître si méprisables à vos yeux que vous nous jugez indignes de présenter des pétitions ou d'adresser des griefs à cette honorable assemblée. » The Humble Petition of divers well-affected women, of the Cities of London and Westminster, p. 1. 
11. «Nous ne nous tiendrons pas tranquilles tant que nous n'aurons pas obtenu de vous que nos époux, nos enfants, nos amis et nos serviteurs ne risquent pas d'être ainsi maltraités, violentés et massacrés selon le bon plaisir de certains. Mais s'il n'y a pour satisfaire votre soif que le sang de ces hommes épris de justice, ces défenseurs inlassables du peuple, alors buvez et étouffez-vous avec notre sang, et nous tomberons ensemble. Emparez-vous encore du sang d'un seul de nos hommes; emparez-vous alors de nous tous. Tuez un seul d'entre nous; alors, tuez-nous tous autant que nous sommes. » The Humble Petition of divers well-affected women, p. 1.

12. "[One should] analyse Leveller presentations of gender as part of the Leveller movement itself, rather than as an episode in women's activism. » HUGHES Ann, «Gender and Politics in Leveller Literature ", in AMUSSEN Susan D. et KISHLANSKY Mark (dir.), Political Culture and Cultural Politics in Early Modern England, Manchester, Manchester University Press, 1995, p. 164.

13. «In their attitude to women the Levellers were ahead of their time. » BRAILSFORD H. N., The Levellers and the English Revolution, $2^{\mathrm{e}}$ éd., Nottingham, Spokesman, 1983, p. 316.

14. FOXLEY Rachel, The Levellers - Radical Political Thought in the English Revolution, Manchester, Manchester University Press, 2013, p. 111.

15. HUGHES Ann, « Gender and Politics in Leveller Literature », p. 163.

16. WINSTANLEY Gerrard, "The New Law of Righteousness ", in The Complete Works of Gerrard Winstanley. CORNS Thomas N., HUGHES Ann et LOEWENSTEIN David (dir.), Oxford, Oxford University Press, 2009, vol. 1, p. 481-2.

17. Le terme « contestataire » désigne ici une manifestation de défiance envers l'ordre établi.

18. Il s'agit de A Perfect Diurnall $\mathrm{n}^{\circ} 300$, p. 2417-18, 2439 (numérotation irrégulière); de The Kingdomes Weekly Intelligencer $n^{\circ} 309$, p. 1337-39 ; et de Perfect Occurrences n¹21, p. 990, 993, 997-8.

19. Voir Perfect Occurrences $n^{\circ} 121$, p. 990.

20. The humble Petition and Representation of severall churches of God in London, commonly, though falsely, called Anabaptists, Londres, 1649. Cette pétition fut intégralement publiée dans Perfect Occurrences n 118, 30 mars - 5 avril 1649, p. 927-8, et dans A Perfect Diurnall n² 297, 2-9 avril 1649, p. 2405-6.

21. Il s'agit de Perfect Occurrences $n^{\circ} 123,4-11$ mai 1649, p. 1023, et de The Kingdomes Weekly Intelligencer $n^{\circ} 310$, 1-8 mai 1649, p. 1351, dont le reportage était plus détaillé. Ni A Perfect Diurnall, ni A Perfect Weekly Account, ou encore The Moderate Intelligencer, n'évoquèrent cette pétition.

22. «Elles ont toujours aimé, et ce depuis le début, se mêler de choses qui ne les regardaient pas; ces poissons chétifs vont bientôt se faire prendre par de petits hameçons. » A Modest Narrative of Intelligence $\mathrm{n}^{\circ}$ 6, 5-12 mai 1649, p. 42.

23. "A gentilhomme leur a signifié que, avec un peu de jugement, elles comprendraient que leur place est à la maison. » The Impartiall Intelligencer $n^{\circ} 10,2-9$ mai 1649, p. 90.

24. « Cela revient à dire : laissons les femmes porter la culotte. [...] Le monde ne peut pas tourner rond quand les femmes se mêlent de politique. [...] Nous voilà dans de beaux draps si les femmes viennent apprendre au Parlement comment faire les lois. » The Impartiall Intelligencer $n^{\circ}$ 8, 18-25 avril 1649, p. 63.

25. «Afin d'empêcher qu'à l'avenir, aucun groupe de royalistes et de mécontents ne puisse se rassembler au nom de ces gens ridicules pour commettre un tort plus grand encore. » Document reproduit dans LUTAUD Olivier, Winstanley - Socialisme et christianisme sous Cromwell, Paris, Didier, 1976, p. 174. Sur Les Bêcheurs et la presse, voir CURELly Laurent , «'Our Digging upon that Common is the Talk of the Whole Land': The Story of the Surrey Diggers as Told by Contemporary Newsbooks", in BANDRY-SCUBBI Anne \& CHARDIN Jean-Jacques (dir.), Diffusion de l'écrit dans le monde anglophone- Spreading the Written Word in the English-Speaking World, Revue de la Société d'Études Anglaises et Américaines XVII-XVIII ${ }^{e}$ siècles, H.S. n², 2010, p. 47-62.

26. Selon Mercurius Pragmaticvs, «they make use of their Tongues (as their best weapon) against Tyranny » (« elles utilisent leur meilleure arme, leur langue, pour combattre la tyrannie »), $n^{\circ} 52$, 
24 avril - 1 mai 1649, n. p. Mercurius Elenticus écrit : " They had put on their best Petticoats and set their Eyes in a posture of temptation " («Elles avaient revêtu leurs plus beaux jupons et faisaient les yeux doux pour mieux séduire $»), n^{\circ} 1,24$ avril - 1 mai 1649, p. 3.

27. Voir MCELLIGOTT Jason, Royalism, Print and Censorship in Revolutionary England, Woodbridge, The Boydell Press, 2007.

28. «Ils vont voir ce que c'est que de se moquer des femmes à propos de leurs tâches domestiques quand il est question pour elles de liberté », Mercurius Pragmaticus (for King Charles II) n³, 1-8 mai 1649, n.p.

29. "Si vous vous contentez de pétitionner, vous prouverez que les mots ne sont que du vent et vous feront le plus grand tort en suscitant une haine plus grande encore chez vos ennemis; par conséquent, faites preuve de courage, agissez, il y a assez de femmes pour détruire Cromwell et ses partisans. [...] Cessez de faire des pétitions, dégainez vos épées, battez-vous pour vos libertés, dûssiez-vous être couvertes de sang jusqu'au menton ", Mercurius Pragmaticvs n52, n. p.

30. Mercurius Militaris, Or The People's Scout, n¹, 17-24 avril 1649, p. 13.

31. "Les femmes avancent en rangs serrés; elles vont mener une deuxième charge contre les députés, armées d'une pétition. [...] Mais Nol [surnom attribué à Cromwell] et ses ânes / Effrayés par ces valeureuses femmes / Prennent leurs jambes à leur cou ", Mercurius Militaris, Or The People's Scout, $n^{\circ} 3$, s.d., p. 32.

32. The Moderate, $\mathrm{n}^{\circ} 43,1-8$ mai 1649 , p. 490-1.

33. Voir HOLSTUN James, Ehud's Dagger - Class Struggle in the English Revolution, London \& New York: Verso, 2000, p. 106-112 ; PEACEY Jason, Print and Public Politics in the English Revolution, op. cit., p. 267-298; PEACEY Jason, "The Parliamentary Context of Political Radicalism in the English Revolution ", in CURELLY Laurent et SMITH Nigel, Radical Voices, Radical Ways - Articulating and Disseminating Radicalism in Seventeenth- and Eighteenth-Century Britain, Manchester, Manchester University Press, 2016, p. 151-169.

\section{RÉSUMÉS}

Cet article a pour objectif d'évaluer l'engagement des épouses des Niveleurs au printemps 1649, après le régicide et la mise en place de la république, engagement qui se manifesta par deux pétitions adressées au Parlement Croupion. Leurs maris avaient été arrêtés après avoir publié un pamphlet jugé séditieux ; aussi leurs femmes demandèrent-elles leur libération, exprimant dans le même temps des revendications qui portaient atteinte à l'ordre patriarcal. La présente contribution analyse également la représentation "médiatique » du combat de ces femmes à travers les comptes rendus qu'en firent les journaux contemporains : journaux acquis à la cause parlementaire, hebdomadaires royalistes et publications radicales. Elle montre comment le soutien qu'apporta une certaine presse aux Niveleuses transforma leur engagement en faveur de leurs époux en une forme de participation politique.

This essay explores the Leveller women's activism as expressed in the two petitions they presented to the Rump Parliament in the spring of 1649 after the regicide and the establishment of the Commonwealth. The Levellers had been arrested and committed to the Tower of London for publishing a pamphlet that was considered seditious. Their wives called for their release while making demands that challenged the patriarchal order. This contribution also looks into the way the contemporary press reported on the Leveller women's fight; the newspapers studied 
here include middle-of-the-road parliamentarian weeklies, royalist "mercuries" as well as radical newsbooks. This essay shows that some of these publications advertised Leveller women's efforts to have their husbands released, thus turning disenfranchised individuals into political activists.

INDEX

Keywords : Levellers, women, English Civil War, press, Rump Parliament, English Revolution Mots-clés : Levellers, femmes, Niveleurs, guerre civile anglaise, presse, révolution anglaise, parlement croupion

\section{AUTEUR}

\section{LAURENT CURELLY}

ILLE, EA 4363, Université de Haute Alsace, 68093 Mulhouse 